Supporting Information

\title{
Self-Powered Smart Arm Training Band Sensor Based on Extremely Stretchable
} Hydrogel Conductors

Feifan Sheng ${ }^{1,2}$, Jia Yi ${ }^{1,2}$, Shen Shen ${ }^{2}$, Renwei Cheng ${ }^{2,3}$, Chuan Ning ${ }^{2,3}$, Liyun $\mathrm{Ma}^{2}$, Xiao Peng ${ }^{2,3}$, Wen Deng ${ }^{1}$, Kai Dong ${ }^{2,3 *}$, Zhong Lin Wang ${ }^{2,3,4,5^{*}}$

1. School of Chemistry and Chemical Engineering, Center on Nanoenergy Research, School of Physical Science \& Technology, Guangxi University, Nanning 530004, P.R. China

2. CAS Center for Excellence in Nanoscience Beijing Key Laboratory of Micro-Nano Energy and Sensor, Beijing Institute of Nanoenergy and Nanosystems, Chinese Academy of Sciences, Beijing 100083, P.R. China

3. School of Nanoscience and Technology, University of Chinese Academy of Sciences Beijing 100049, P.R. China

4. CUSTech Institute, Wenzhou, Zhejiang, 325024, China.

5. School of Material Science and Engineering, Georgia Institute of Technology Atlanta, GA 30332, USA

* Corresponding authors:

dongkai@binn.cas.cn (K. Dong), zhong.wang@mse.gatech.edu (Z. L. Wang). 
Movie S1 (.mp4 format). True super tensile stress-strain curve of SA-Zn hydrogel.

Movie S2 (.mp4 format). Conductive of stretched SA-Zn hydrogel.

Movie S3 (.mp4 format). Lighting LEDs with the energy converted from hand flapping by a SA-Zn hydrogel TENG.

Movie S4 (.mp4 format). Application of an arm training band with the energy converted from hand stretching by a SA-Zn TENG.

Figure S1. FTIR spectra of the SA-Zn hydrogel at different proportions of AM and AA.

Figure S2. Performance comparison of different content of SA.

Figure S3. Performance comparison of different content of initiator APS.

Figure S4. Performance comparison of different content of $\mathrm{ZnSO}_{4} \cdot 7 \mathrm{H}_{2} \mathrm{O}$.

Figure S5. The elastic modulus of the SA-Zn hydrogel at different proportions of AA and AM.

Figure S6. Electrochemical impedance spectroscopy of SA-Zn hydrogel.

Figure S7. Differential scanning calorimetry (DSC) analysis of SA-Zn hydrogel.

Figure S8. Thermogravimetric analysis (TGA) of SA-Zn hydrogel.

Figure S9. Water retaining property of SA-Zn hydrogel.

Figure S10. Mechanical properties of the hydrogel after $72 \mathrm{~h}$ at room temperature.

Figure S11. Tensile stress-strain curves at different strain rates.

Figure S12. Correlation between Young's modulus and strain rate.

Figure S13. The working mechanism of the SH-TENG smart training band in single electrode mode.

Figure S14. The $\mathrm{V}_{\mathrm{OC}}$ of SH-TENG under different strains.

Figure S15. The relationship between power density and external load resistance with an external load resistance from $10^{4}$ to $7 \times 10^{9} \Omega$ under different strains.

Figure S16. Electrical properties of the hydrogel after $72 \mathrm{~h}$ at room temperature.

Figure S17. The stability of SH-TENG under 20 days. 


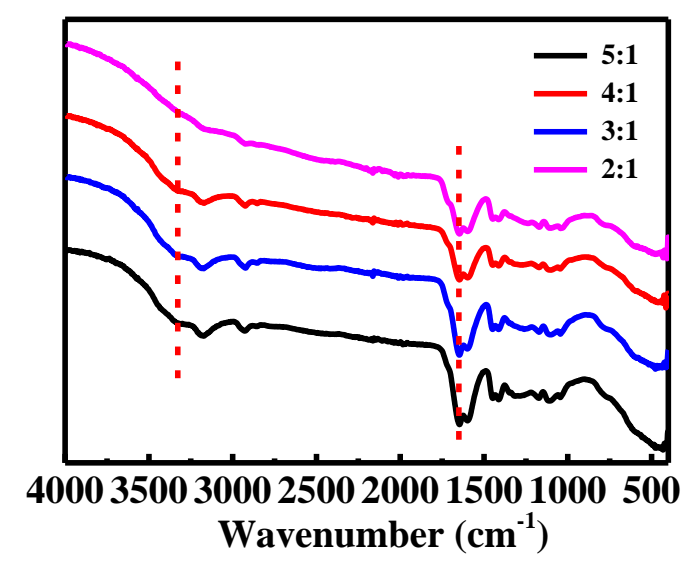

Figure S1. FTIR spectra of the SA-Zn hydrogel at different proportions of AM and AA.

Varying the amount of acrylamide can change the FT-IR spectra. Figure S1 shows FTIR spectra of the SA-Zn hydrogel at different proportions of AM and AA. The difference in the mechanical properties is partly caused by hydrogen bonds, so the FT-IR spectrum changes when the content of acrylamide changes. It can be seen from the figure that the characteristic absorption peak of the amide group appears at $3328 \mathrm{~cm}^{-1}$, and it increases with the increase of the amount of acrylamide, and the characteristic absorption peak of the carboxylic acid group at $1650 \mathrm{~cm}^{-1}$ also increases. The SA-Zn hydrogel can obtain high characteristic absorption peaks of the amide group and the carboxylic acid group with AA and AM feed ratio of 1:3, and the group peak is higher than 1:4. It can be confirmed that the FTIR spectra of the SA-P(AM-AA) double network copolymer increases in intensity with increasing the amount of acrylamide within a certain range.

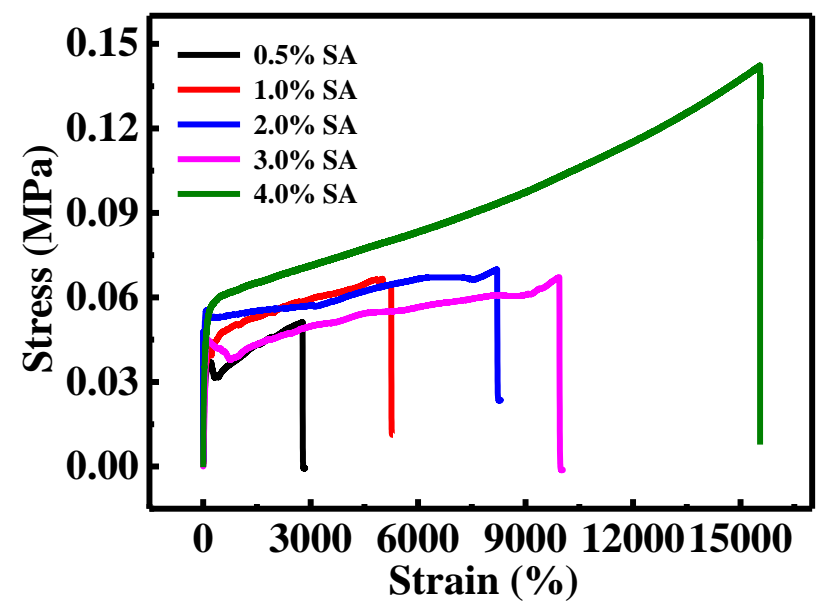

Figure S2. Performance comparison of different content of SA. 
In this study, five hydrogels with different SA contents were prepared. From Figure S2, it can be found that as the SA concentration gradually increases from $0.5 \%$ to $4 \%$, the hydrogel strength has been increasing. The tensile strength at break reaches the maximum value $(0.15 \mathrm{MPa})$ when the $\mathrm{SA}$ concentration is $4 \%$. However, the corresponding elongation at break reaches the maximum value exceeding $10000 \%$. In summary, as well as the optimal ratio of AA and AM, it shows that the high mechanical properties of the hydrogel do not completely depend on the close cross-linking of the two-layer network, but rather rely on the mutual synergy of the two-layer network. ${ }^{1}$

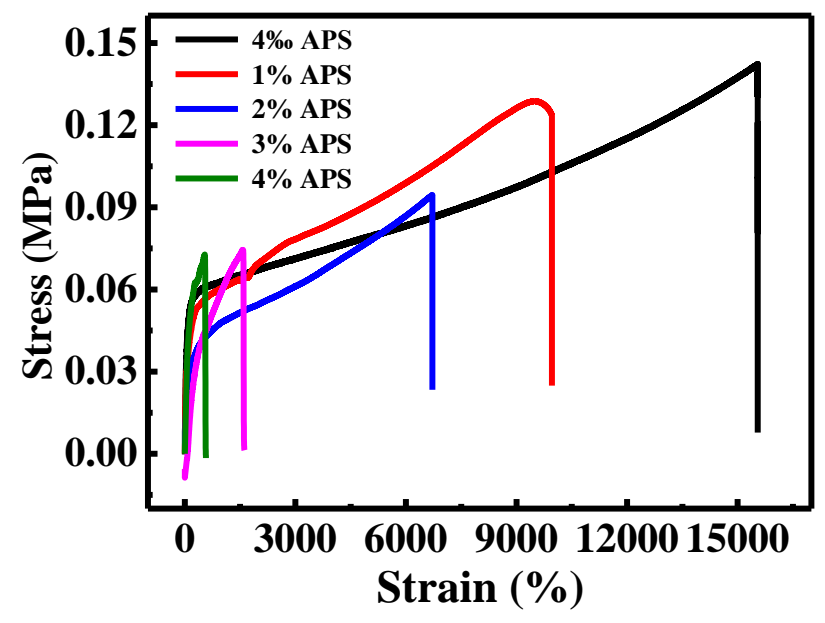

Figure S3. Performance comparison of different content of initiator APS.

The amount of the initiator directly affects the speed of the polymerization reaction and the molecular weight of the polymerization product. For a common free radical polymerization reaction, an increase in the amount of the initiator will accelerate the reaction speed. When the content of initiator is too low, less free radicals are generated, making the polymerization reaction difficult or requiring a long time to react, and the monomer conversion rate is low, the resulting product has a low degree of crosslinking and cannot form a good three-dimensional network Structure, resulting in poor mechanical properties, but when the content of the initiator is too high, the reaction speed of the system is too fast, difficult to control, easy to produce explosions, the reaction temperature cannot be controlled, and the product will also cause partial selfcrosslinking. The synthetic hydrogel has relatively low molecular weight and high 
brittleness, resulting in worse mechanical properties. It can be seen from Figure S3 that when the amount of initiator is $0.4 \%$, the mechanical properties of the composite material are optimal.

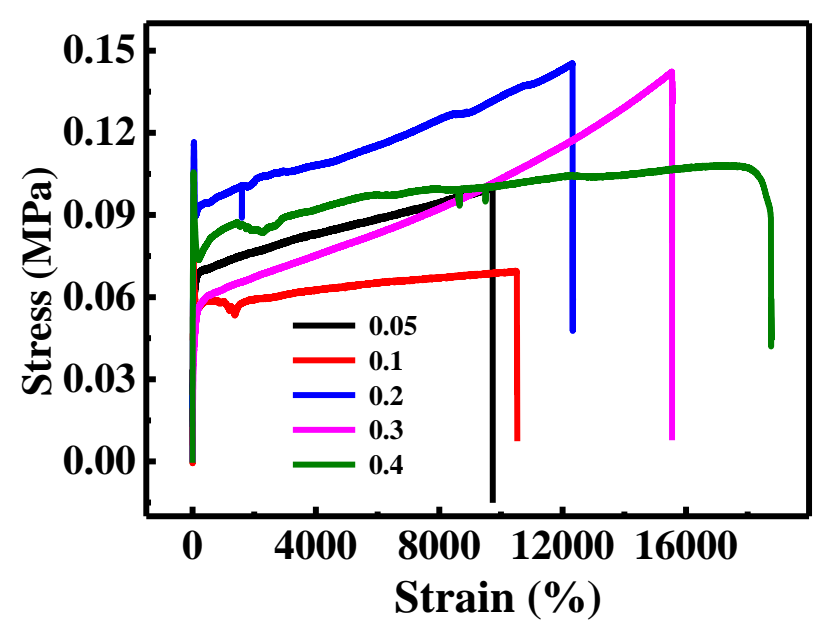

Figure S4. Performance comparison of different content of $\mathrm{ZnSO}_{4} \cdot 7 \mathrm{H}_{2} \mathrm{O}$.

The excellent mechanical properties of SA-Zn hydrogels are mainly due to dynamic interactions. In order to study the effect of dynamic interaction on the mechanical properties, SA-Zn hydrogels with different $\mathrm{ZnSO}_{4} \cdot 7 \mathrm{H}_{2} \mathrm{O}$ contents were prepared as control samples, the mechanical properties are shown in Figure S4. It can be clearly seen from the figure that as the content of $\mathrm{ZnSO}_{4} \cdot 7 \mathrm{H}_{2} \mathrm{O}$ increases, the mechanical properties of SA-Zn hydrogels gradually improve and eventually weaken. When the mass of $\mathrm{ZnSO}_{4} \cdot 7 \mathrm{H}_{2} \mathrm{O}$ increased from $0.05 \mathrm{~g}$ to $0.3 \mathrm{~g}$, more dynamic interactions are formed between $\mathrm{Zn}$ (II) and the ammonium/carboxyl groups in the polymer molecular chain. The tensile stress increases from $0.06 \mathrm{MPa}$ to $0.15 \mathrm{MPa}$, and the tensile strain expands from $9000 \%$ to $15000 \%$. The results indicate that the dynamic interaction between $\mathrm{Zn}$ (II) and the ammonium/carboxyl group in the polymer molecule can increase the mechanical properties of SA-Zn hydrogel. ${ }^{2,3}$ These functions provide the super-stretching and self-recovery ability of SA-Zn hydrogel. In addition, the maximum elongation at break $(18000 \%)$ is reached when the mass of zinc sulfate is $0.4 \mathrm{~g}$, while the tensile strength at break is only $0.105 \mathrm{MPa}$. This may be because when the $\mathrm{Zn}^{2+}$ concentration is low, the mechanical properties of the hydrogel will be poor due to 
insufficient cross-linking, while too high a $\mathrm{Zn}^{2+}$ concentration will cause uneven crosslinking and make the mechanical properties of the hydrogel worse or unstable. Therefore, we chose $0.3 \mathrm{~g} \mathrm{ZnSO} \cdot 7 \mathrm{H}_{2} \mathrm{O}$ in the later experiments.

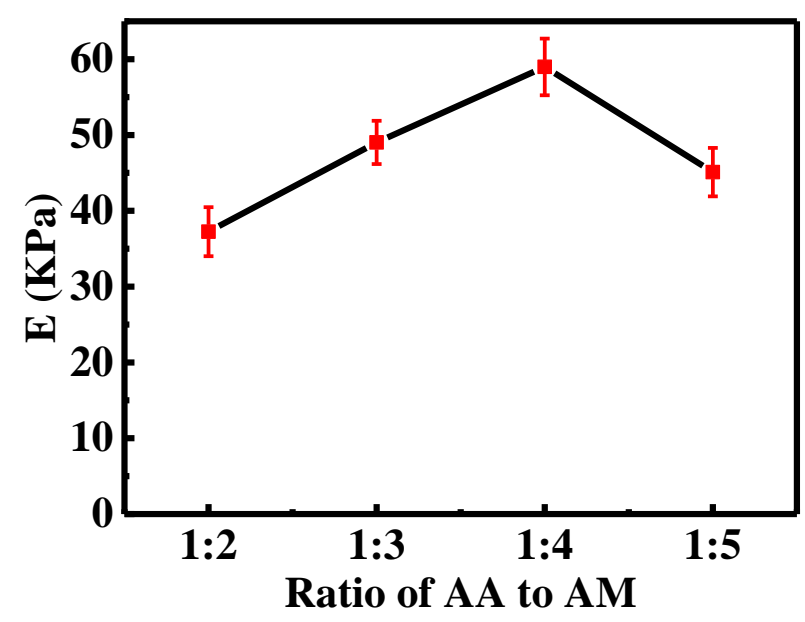

Figure S5. The elastic modulus of the SA-Zn hydrogel at different proportions of AA and AM.

The elastic modulus of the SA-Zn hydrogel at different proportions of AA and AM are shown in Figure S5. Elastic modulus is calculated from the stress-strain curves (Figure $2 \mathrm{~d})$. When the ratio of AA to AM is $1: 2,1: 3,1: 4,1: 5$, the elastic modulus is $37.25 \mathrm{KPa}$, 49.02 KPa, $58.98 \mathrm{KPa}$, and 45.1 KPa, respectively. As the AM content rises, the elastic modulus of SA-Zn hydrogel shows an increase followed by a decrease. As the ratio rising over $4: 1$, the modulus jumped from $58.98 \mathrm{KPa}$ to $45.1 \mathrm{KPa}$ at a $5: 1$ ratio. This abnormal phenomenon is probably caused by acrylamide changing the hydrogen bond, and at the same time is caused by the increasing the cross-linking site density and dynamic equilibrium of zinc ions with the two polymer chains. 


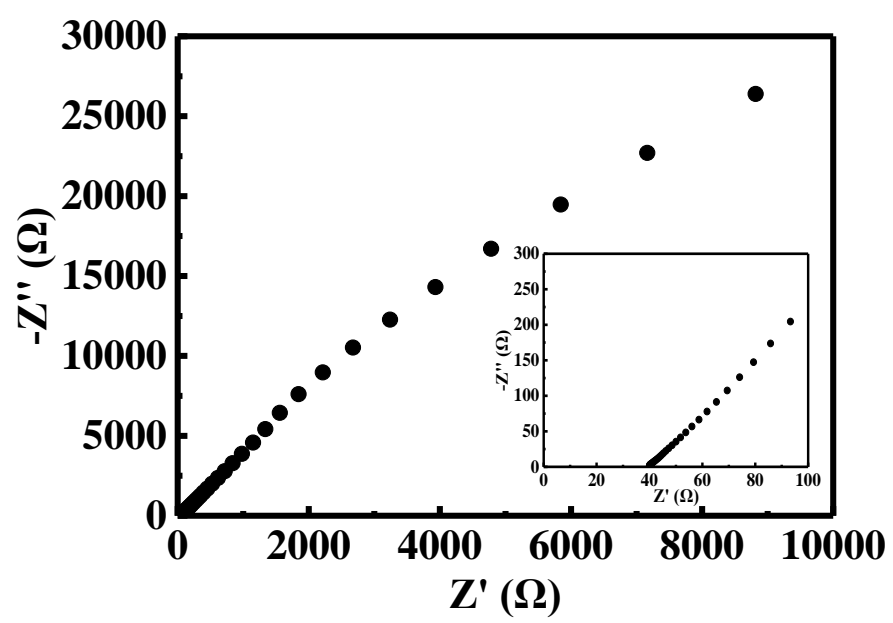

Figure S6. Electrochemical impedance spectroscopy of SA-Zn hydrogel.

As shown in Figure S6, we used a two-electrode system (stainless steel foil // SA-Zn hydrogel // stainless steel foil) to measure electrochemical impedance spectroscopy (EIS). When the size of the hydrogel is $8.58 \mathrm{~mm} \times 9.35 \mathrm{~mm} \times 1.08 \mathrm{~mm}$, as shown in the inset of Figure S6, the resistance is $40 \Omega$. The ionic conductivity of the SA-Zn hydrogel can be calculated according to the following formula:

$$
\begin{aligned}
& \kappa=\frac{1}{\rho} \\
& R=\rho \frac{l}{A}
\end{aligned}
$$

Where R $(\Omega)$ is the resistance of the hydrogel, $1(\mathrm{~m})$ is the length of the hydrogel and $\mathrm{A}\left(\mathrm{m}^{2}\right)$ is the cross-sectional area of the hydrogel. $\rho\left(\Omega \cdot \mathrm{m}^{-1}\right)$ and $\kappa\left(\mathrm{S} \cdot \mathrm{m}^{-1}\right)$ represent the resistivity and conductivity of the hydrogel, respectively. ${ }^{4}$ 


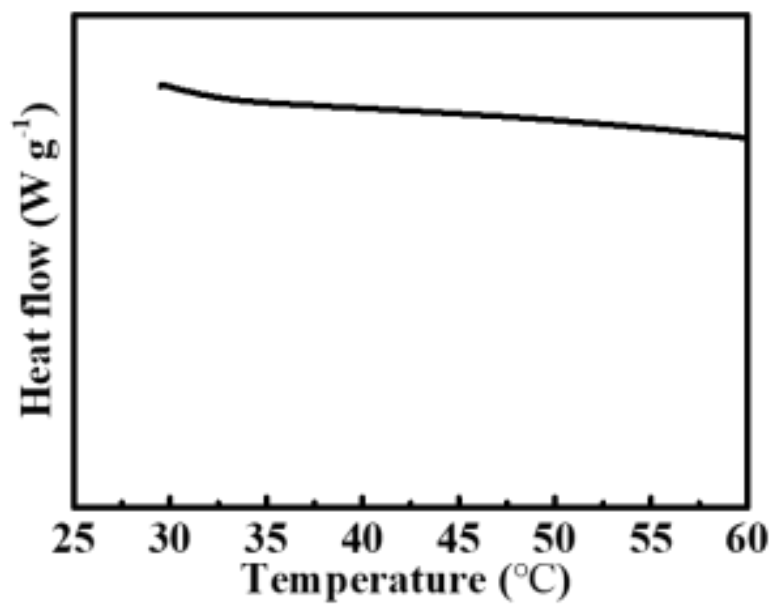

Figure S7. Differential scanning calorimetry (DSC) analysis of SA-Zn hydrogel.

As shown in Figure S7, the SA-Zn hydrogel indicate that there was no characteristic free water peak in the heating process has been observed in the DSC curve from room temperature to $60{ }^{\circ} \mathrm{C}$, indicating that the SA-Zn hydrogel has a good water retention ability. $^{5}$

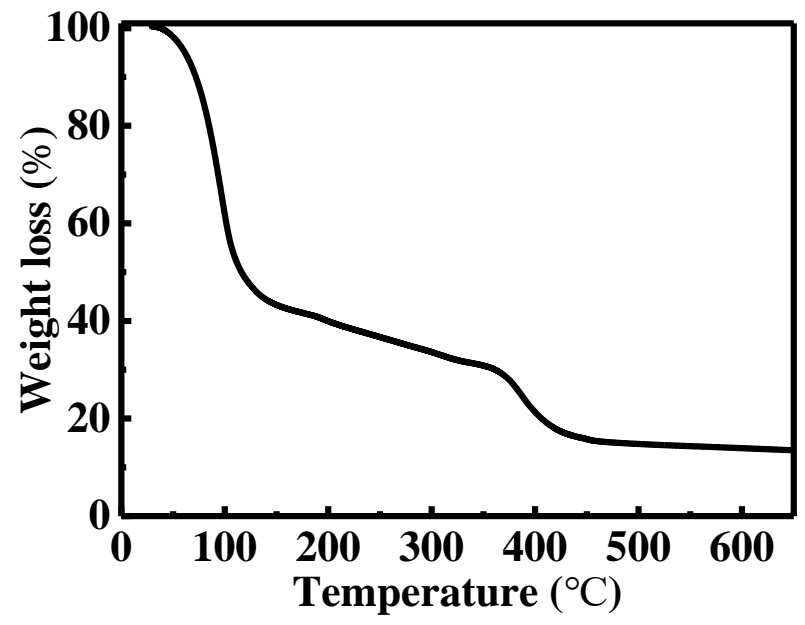

Figure S8. Thermogravimetric analysis (TGA) of SA-Zn hydrogel.

The thermal stability of the SA-Zn hydrogel was investigated as a function of temperature from 25 to $650^{\circ} \mathrm{C}$ in an argon atmosphere. As shown in Figure S8, the SA$\mathrm{Zn}$ hydrogel showed four stages of decomposition processes. It can be seen from the figure that the first weight loss stage of SA-Zn hydrogel in the temperature range of 
room temperature to $112{ }^{\circ} \mathrm{C}$ was $49.49 \%$, mainly due to the volatilization of free water in the hydrogel and the loss of bound water in the SA molecule. The second weight loss was $8.44 \%$ at $186{ }^{\circ} \mathrm{C}$. The weight loss was smaller in this temperature range, mainly because the molecular chains of the PAM-co-PAA hydrogel began to move and the amide bond of the crosslinker began to break. The third weight loss was around $357^{\circ} \mathrm{C}$, the bound water inside the hydrogel began to volatilize, the carboxyl group in acrylic acid began to degrade, and the SA molecular chain was broken and decomposed into a stable intermediate product and partially carbonized. In the fourth weight loss at $437^{\circ} \mathrm{C}$, acrylamide began to degrade, C-C single bonds began to break, and the hydroxyl and carboxyl groups of sodium alginate were removed and completely carbonized. The weight loss was $13.45 \%$, and the final residual ash content was $13.62 \%$.

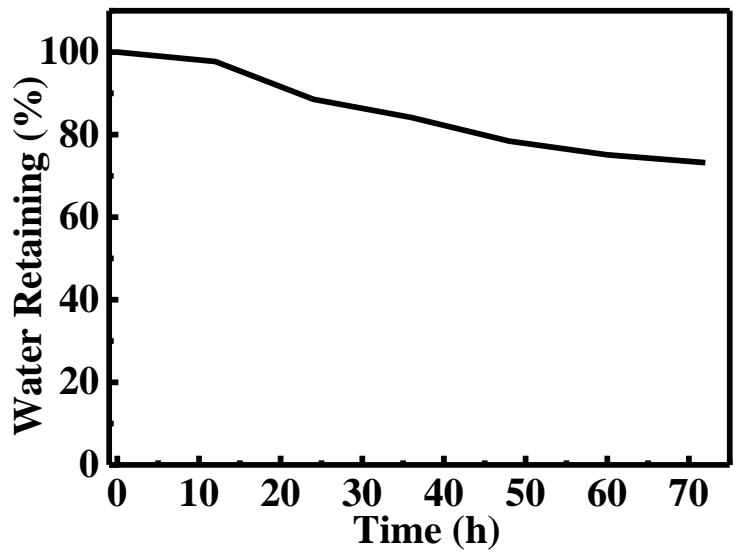

Figure S9. Water retaining property of SA-Zn hydrogel.

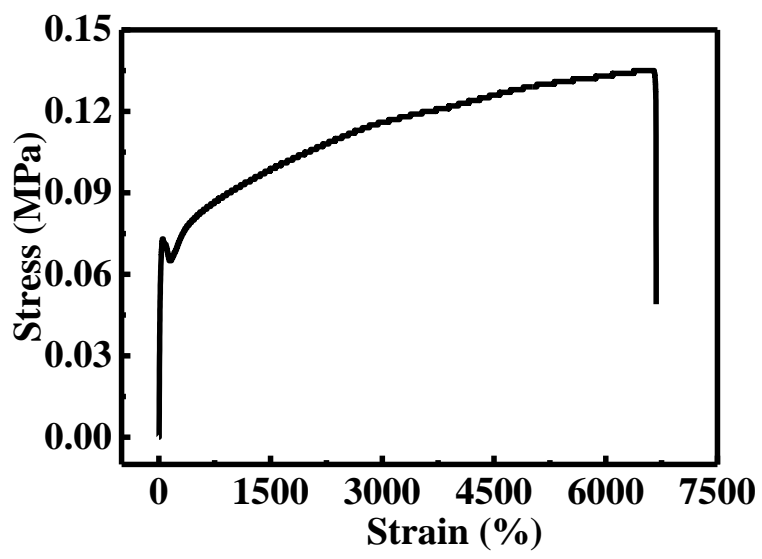


Figure S10. Mechanical properties of the hydrogel after $72 \mathrm{~h}$ at room temperature.

The SA-Zn hydrogel has good water retaining ability. After being put at $25^{\circ} \mathrm{C}$ for $72 \mathrm{~h}$, it still can maintain nearly $73 \%$ water content (Figure S9). These good properties demonstrate that the SA-Zn hydrogel possesses good reusability and stability. As can be seen from Figure S10, the hydrogel is placed for $72 \mathrm{~h}$, and its stretchable performance is still excellent.

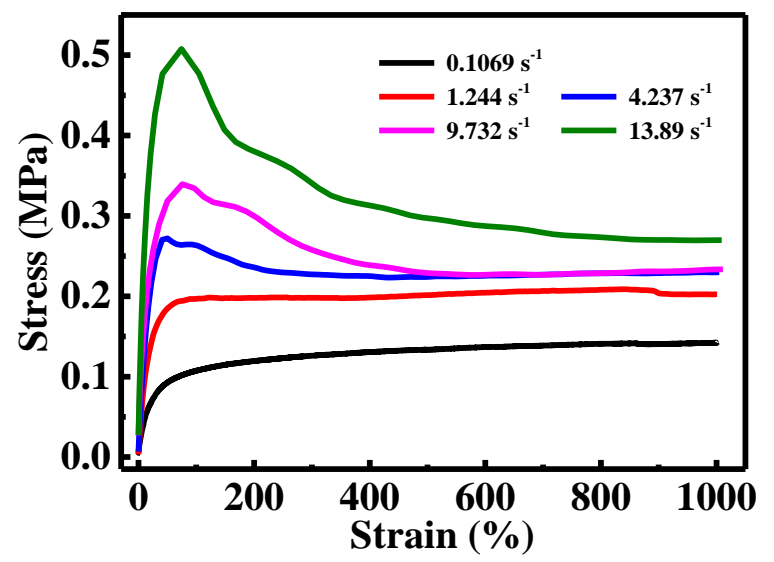

Figure S11. Tensile stress-strain curves at different strain rates.

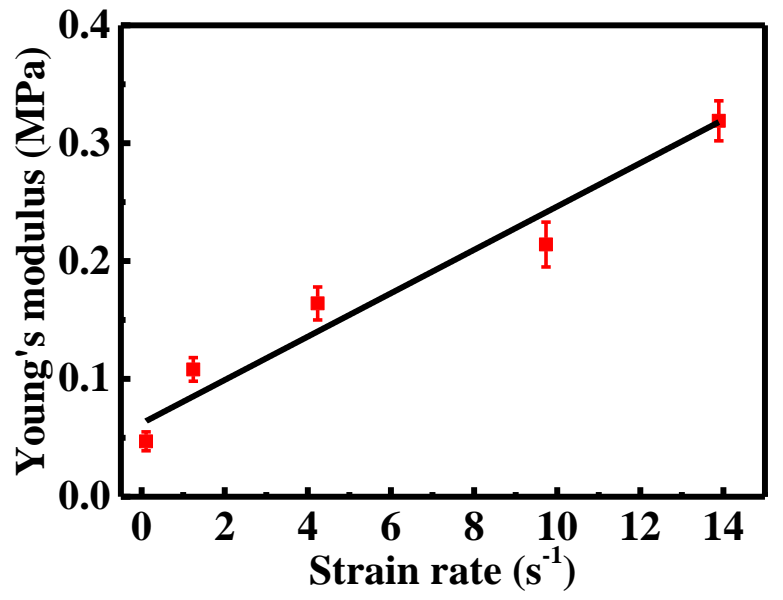

Figure S12. Correlation between Young's modulus and strain rate. 
Because of the relaxation of dynamic hydrogen bonds, the increase in deformation rate entails a faster dissociation process of reversible cross-links and requires a higher mechanical strength, leading to the increase in the modulus and toughness. In this regard, the mechanical properties of composite hydrogels can be viewed as apparent parameters that are significantly dependent on the deformation conditions, such as temperature and stretch rate. Because we restrict the scope of current effort in discussing the deformation response of gels under isothermal condition and neglect possible water diffusion in the gels, we attempt to model the following mechanical behavior at different strain rates with the rate ranging over near 3 orders of magnitude from 0.1069 to $13.89 \mathrm{~s}^{-1}$ (Figure S11). The larger strain rate in the experiment is to take into account the rapid speed of stretching in the later practical application.

Within $1000 \%$ of our required strain, the Young's modulus exhibits a certain strain rate dependent behavior (Figure S12). Within the test strain rate, even if the strain rate is very high, the Young's modulus presents increasing phenomenon. The excellent stretching flexibility of the SA-Zn hydrogel can be attributed to the dynamic interactions of the coordination interaction and electrostatic interaction, which are relatively weak but still stronger than a hydrogen bond. When the SA-Zn hydrogel was stretched to a certain length, the tensile stress increases rapidly with tensile strain in a linear relationship, exhibiting obvious elastic deformation. During the stretching process, the molecular chain entanglements in the frame structure are broken and the molecular chains are orientated in the direction of the external force. Therefore, the stretching process exhibit large stress, but a small tensile strain. When the tensile strain is greater than a certain value, the tensile stress and strain still maintain a good linear relationship, but with a certain hysteresis. By comparison, the tensile strain below a certain value shows a large tensile strain range with small stress changes, mainly due to the dynamic interactions. After the orientation of the molecular chain, the dynamic interaction plays an important role in connecting the molecular chain to expand the tensile strain. The Zn (II) serve as a bridge in the frame of the hydrogel to connect different molecular chains together. During the stretching process, the dynamic 
interactions are broken by the external force and quickly rebuilt again between the molecular chains to make them unfold and slide, thus maintaining the stable connection and effectively expanding the tensile strain. The formed double-network structure and dynamic interactions of the SA-Zn hydrogel ensure its outstanding mechanical property.

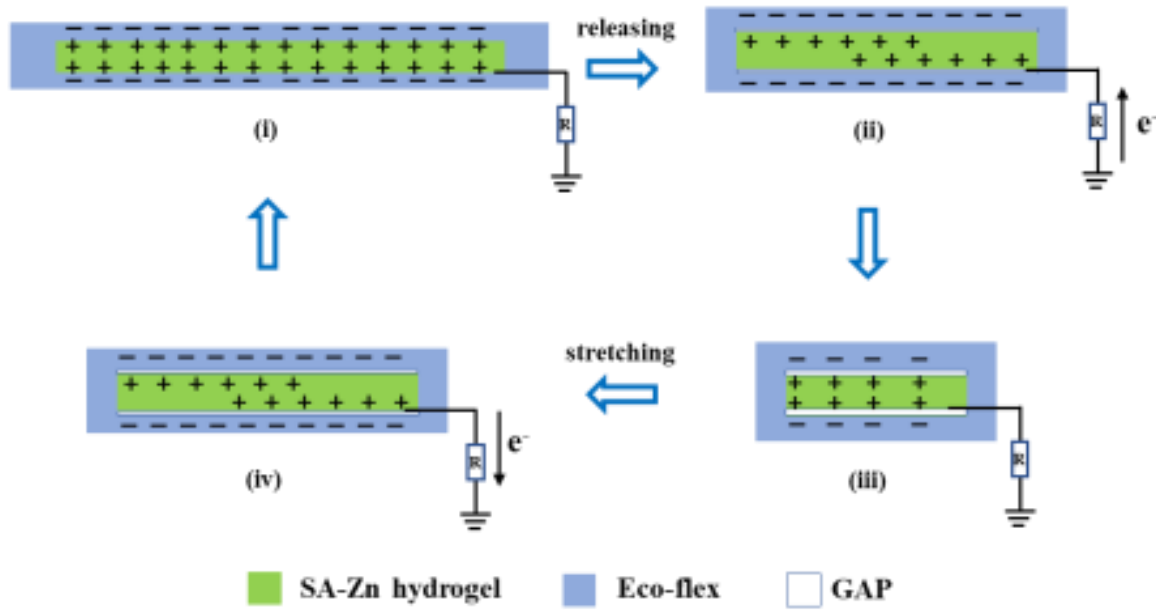

Figure S13. The working mechanism of the SH-TENG smart training band in single electrode mode.

Since the preparation of smart training band, we did not used further treatment to the hydrogel surface and silicone surface, thus the boundary between the two materials may not be completely adhered, so there is a small gap between the hydrogel and the package layer during the formation of the device. The power generation layer and the conductive layer have a contact separation process during the stretching process, which is caused by the coupling effect of triboelectrification and electrostatic induction. The working mechanism of the SH-TENG smart training band is shown in Figure S13. Once the elastic band is stretch to the maximum, the hydrogel is completely contacted with the silicone rubber layer, and a positive charge is generated at the interface. The silicone rubber produces the same amount of negative charges of opposite polarity. At this time, due to the frictional charges are completely balance, no electrons flow in the external circuit, as shown in Figure S13 (i). When the tensile is released, the ecoflex and hydrogel are moved upward, and the positive charges of conductive hydrogel reduce to compensate for the electrostatic charge on the surface of the silicone rubber, balancing the negative charge and reaching an electrical equilibrium state. During this period, 
electrons flow from the copper conductive wire to the hydrogel, generating a macroscopic but instantaneous current signal (Figure S13, from (i) to (ii)). When the stretching state is completely released, ecoflex moves downwards and approaches the hydrogel, the entire process is reversed (Figure S13 (ii)). The positive charges induced on the copper decrease, leading to the electrons flowing in the opposite direction (Figure S13, from (iii) to (iv)). The SH-TENG continuously generates alternating current by repeated stretching and releasing results in periodic operation of ecoflex and hydrogel contact separation process.

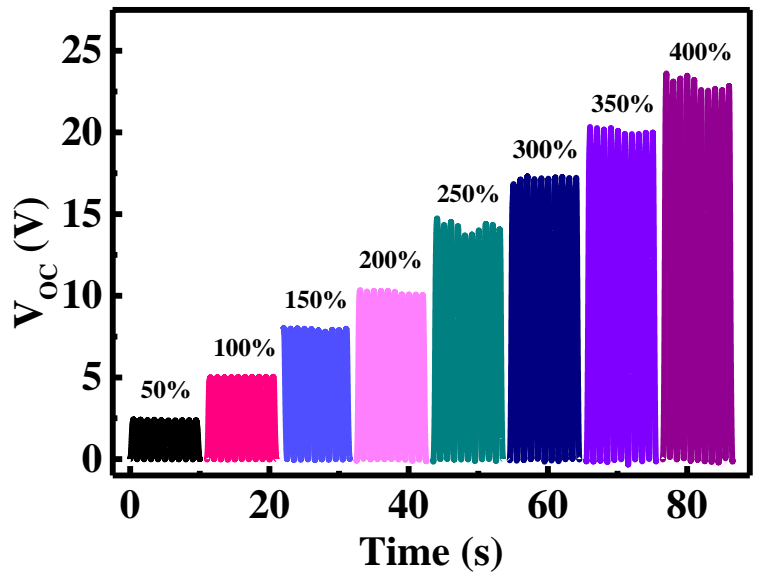

Figure S14. The VOC of SH-TENG under different strains.

The deformation and electricity generation process for the stretching deformation that is explored. It can be seen from Figure S14 that the electrical output increases with the increase of the stretch length, which may attribute to the increase of the distance of the gap between hydrogel and ecoflex during the stretching process. 


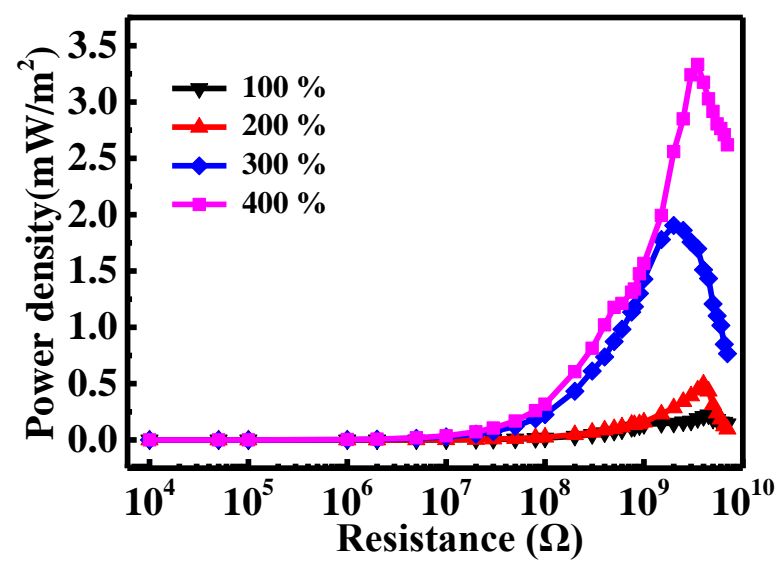

Figure S15. The relationship between power density and external load resistance with an external load resistance from $10^{4}$ to $7 \times 10^{9} \Omega$ under different strains.

As shown in Figure S15, in order to evaluate the effective output power of SH-TENG in different stretched states of $100-400 \%$, the output power density in series with various external resistors $\left(10^{4}-7 \times 10^{9} \Omega\right)$ is studied. It is found that the electrical output of SH-TENG is enhanced with increasing the strains, verifying that the more strains there are, the higher electrical output will be, which can be explained that the smaller the gap between the outer ecoflex and the conductive layer hydrogel.

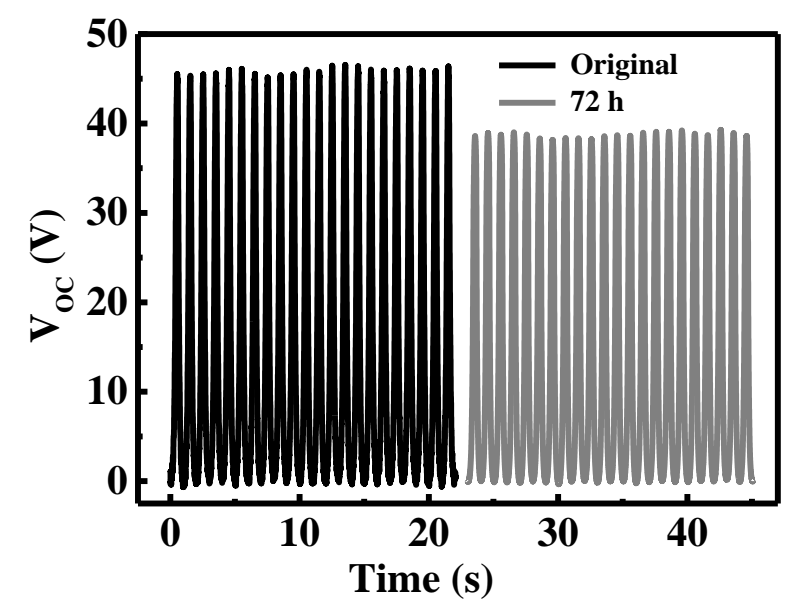

Figure S16. Electrical properties of the hydrogel after $72 \mathrm{~h}$ at room temperature. 


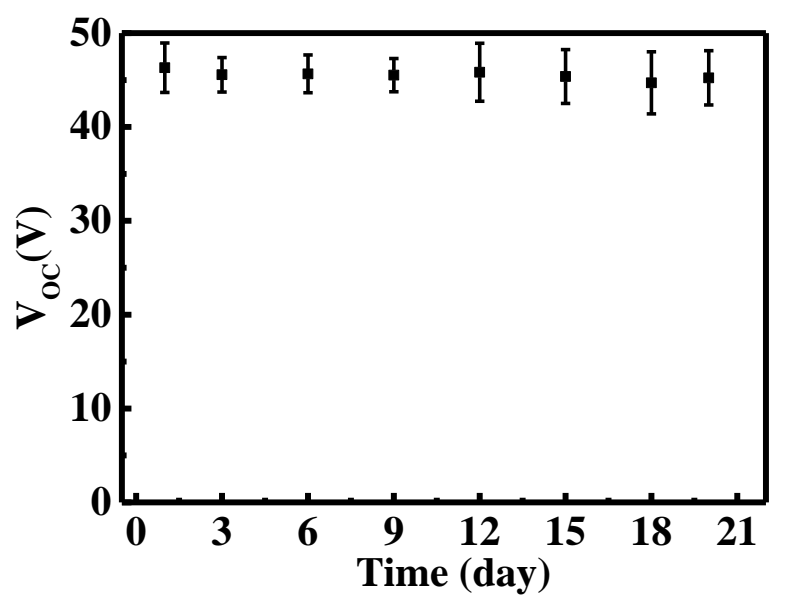

Figure S17. The stability of SH-TENG under 20 days.

According to the water retaining data, we put the hydrogel at room temperature for 3 days, and the TENG electrical properties after packaging the hydrogel were tested (Figure S16). It still can maintain nearly $85 \%$ to its original output performance, verifying the good properties of the SA-Zn hydrogel again. As shown in Figure S17, SH-TENG can keep to its original output performance without significant degradation when the hydrogel packaged with ecoflex under 20 days. This clearly shows that the SH-TENG prepared after direct encapsulation of the hydrogel has excellent stability. 


\section{REFERENCES}

(1) Jalababu, R.; Veni, S. S.; Reddy, K. V. N. S. Synthesis and Characterization of Dual Responsive Sodium Alginate-G-Acryloyl Phenylalanine-Poly N-Isopropyl Acrylamide Smart Hydrogels for the Controlled Release Of Anticancer Drug. J. Drug Deliv. Sci. Tec. 2018, 44, 190-204.

(2) Huang, H.; Han, L.; Li, J.; Fu, X.; Wang, Y.; Yang, Z.; Xu, X.; Pan, L.; Xu, M. Super-Stretchable, Elastic and Recoverable Ionic Conductive Hydrogel for Wireless Wearable, Stretchable Sensor. J. Mater. Chem. A 2020, 8, 10291-10300.

(3) Huang, H.; Han, L.; Fu, X.; Wang, Y.; Yang, Z.; Pan, L.; Xu, M. Multiple Stimuli Responsive and Identifiable Zwitterionic Ionic Conductive Hydrogel for Bionic Electronic Skin. Adv. Electron. Mater. 2020, 6, 2000239.

(4) Han, L.; Huang, H.; Fu, X.; Li, J.; Yang, Z.; Liu, X.; Pan, L.; Xu, M. A Flexible, High-Voltage and Safe Zwitterionic Natural Polymer Hydrogel Electrolyte for High-Energy-Density Zinc-Ion Hybrid Supercapacitor. Chem. Eng. J. 2020, 392, 123733.

(5) Parida, K.; Thangavel, G.; Cai, G.; Zhou, X.; Park, S.; Xiong, J.; Lee, P. S. Extremely Stretchable and Self-Healing Conductor Based on Thermoplastic Elastomer for All-Three-Dimensional Printed Triboelectric Nanogenerator. Nat. Commun. 2019, 10, 2158. 